\title{
BMJ Open Children and Young People's Health Partnership Evelina London Model of Care: economic evaluation protocol of a complex system change
}

\author{
Marina Soley-Bori (D) , ${ }^{1}$ Raghu Lingam, ${ }^{2}$ Rose-Marie Satherley, ${ }^{3}$ Julia Forman, ${ }^{4}$ \\ Lizzie Cecil (10) , Julia Fox-Rushby, ${ }^{5,6}$ Ingrid Wolfe ${ }^{4}$
}

To cite: Soley-Bori M, Lingam R, Satherley R-M, et al. Children and Young People's Health Partnership Evelina London Model of Care: economic evaluation protocol of a complex system change. BMJ Open 2021;11:e047085. doi:10.1136/ bmjopen-2020-047085

- Prepublication history for this paper is available online. To view these files, please visit the journal online (http://dx.doi org/10.1136/bmjopen-2020047085).

JF-R and IW are joint last authors.

Received 19 November 2020 Accepted 18 August 2021

Check for updates

(C) Author(s) (or their employer(s)) 2021. Re-use permitted under CC BY-NC. No commercial re-use. See rights and permissions. Published by BMJ.

For numbered affiliations see end of article.

Correspondence to

Dr Marina Soley-Bori;

marina.soley_bori@kcl.ac.uk

\section{ABSTRACT}

Introduction The Children and Young People's Health Partnership (CYPHP) Evelina London Model of Care is a new approach to integrated care delivery for children and young people (CYP) with common health complaints and chronic conditions. CYPHP includes population health management (services shaped by data-driven understanding of population and individual needs, applied in this case to enable proactive case finding and tailored biopsychosocial care), specialist clinics with multidisciplinary health teams and training resources for professionals working with CYP. This complex health system strengthening programme has been implemented in South London since April 2018 and will be evaluated using a cluster randomised controlled trial with an embedded process evaluation. This protocol describes the within-trial and beyond-trial economic evaluation of CYPHP.

Methods and analysis The economic evaluation will identify, measure and value resources and health outcome impacts of CYPHP compared with enhanced usual care from a National Health Service/Personal Social Service and a broader societal perspective. The study population includes 90000 CYP under 16 years of age in 23 clusters (groups of general practitioner (GP) practices) to assess health service use and costs, with more detailed costeffectiveness analysis of a targeted sample of 2138 CYP with asthma, eczema or constipation (tracer conditions). For the cost-effectiveness analysis, health outcomes will be measured using the Paediatric Quality of Life Inventory and quality-adjusted life years (QALYs) using the Child Health Utility 9 Dimensions (CHU-9D) measure. To account for changes in parental well-being, the Warwick-Edinburg Mental Well-being Scale will be integrated with QALYs in a cost-benefit analysis. The within-trial economic evaluation will be complemented by a novel long-term model that expands the analytical horizon to 10 years. Analyses will adhere to good practice guidelines and National Institute for Health and Care Excellence public health reference case.

Ethics and dissemination The study has received ethical approval from South West-Cornwall and Plymouth Research Ethics Committee (REC Reference: 17/SW/0275). Results will be submitted for publication in peer-reviewed journals, made available in briefing papers for local decision-makers, and provided to the local community
Strength and limitations of this study

Robust study design: Children and Young People's Health Partnership (CYPHP) will be evaluated using a cluster randomised controlled trial with an embedded process evaluation.

- Multiple analytical perspectives: both the National Health Service and Personal Social Services perspective and a societal perspective, accounting for costs falling on parents and schools, will be adopted.

- Long analytical horizon: the within-trial economic evaluation will be complemented by a novel longterm model that expands the analytical horizon to 10 years.

- Impact of COVID-19 on CYPHP service delivery: differences in the frequency and duration of each CYPHP component before and after COVID-19 may be observed, which will be assessed in sensitivity analyses.

- Measurement of intervention effects: the intensity of the different intervention components may have varied across general practitioner practices, and the measurement of health effects with the Child Health Utility 9 Dimensions for children below 5 may lack reliability.

through website and public events. Findings will be generalisable to community-based models of care, especially in urban settings.

Trial registration number NCT03461848.

\section{INTRODUCTION}

In 2018, nearly 1400 excess child deaths occurred in the UK compared with Sweden, adjusting for population size. ${ }^{12}$ The UK fares worse than other high-income countries in chronic disease management too. Only 16\% of young people in the UK with type 1 diabetes had a glycated haemoglobin A1c under $7.5 \%$, whereas in Germany and Austria, this standard was met for $34 \%$ of young people..$^{3-5}$ Poor chronic disease management results in worse health-related quality of $\operatorname{life}^{67}$ and 
in higher emergency room visits and hospitalisations, which are key healthcare cost drivers. ${ }^{5-11}$ Beyond direct medical costs, poorly controlled chronic conditions result in time lost from school and employment, placing a significant burden on families. For example, the overall cost of caring for children with asthma aged 1-5years in the 12 months following attendance at hospital for wheeze or asthma is estimated to be $£ 14.53$ million.$^{12}$

Ensuring good health in childhood is a public health priority both as a rights-based principle ${ }^{13}$ and for the health, social and economic consequences in adulthood. ${ }^{14} 15$ Notwithstanding the current pandemic, the UK paediatric healthcare delivery model-originally designed to treat acute conditions through high-intensity specialist and inpatient services-now needs to address chronic healthcare needs and emphasise preventive care. Previous efforts to integrate care for children and young people (CYP) with ongoing conditions have shown potential for improving quality of life and reducing costs, but evidence is limited. ${ }^{16}$

The Children and Young People's Health Partnership (CYPHP) Evelina London Model of Care is an innovative approach to integrated healthcare delivery. It was implemented in April 2018 in two London boroughs (Lambeth and Southwark) where Accident \& Emergency care (A\&E) attendance for children aged 0-4 years and hospital admissions related to asthma were $16 \%$ and $25 \%$ higher than the national average, respectively. ${ }^{17}$ The CYPHP model aims to strengthen the health system by bridging the gap between primary and secondary care as well as physical and mental health, and links healthcare with local efforts to tackle the socioeconomic determinants of health. Through coordinated, early intervention and biopsychosocial care delivered in primary care and community settings, CYPHP has been developed to promote better healthcare and selfmanagement for CYP with common health complaints and chronic conditions. ${ }^{18} 19$ The concept of biopsychosocial care follows many of the tenets of patient centred care as outlined by Tramonti and colleagues ${ }^{20}$; however, we use a more specific term to describe the model in greater detail.

CYPHP will be implemented across Southwark and Lambeth in two stages. The staged implementation offers a platform for an opportunistic clustered randomised controlled trial study design for rigorous evaluation purposes, running alongside a service evaluation reporting regularly to a partnership board of commissioner, provider, community organisations and researchers. In the first CYPHP deployment stage (approximately 3 years), general practices were randomised to either CYPHP (intervention) or enhanced usual care (EUC-control). CYPHP includes the EUC components but also in-reach clinics, lunch-and-learn sessions, specialist nurse-led services, population health management, specialist team training and multidisciplinary team case planning. After 3 years, CYPHP will be implemented in all of the practices.
The aims of the embedded economic evaluation are, first, to assess the impact of CYPHP compared with EUC on patient-level healthcare costs from a National Health Service (NHS) and Personal Social Service (PSS) perspective for the entire trial population. PSS includes a range of services provided by local authorities for vulnerable groups, including the mentally and physically disabled, older people and neglected children. The second aim, among children with specific targeted tracer conditions, is to compare costs and health outcomes and establish the cost-effectiveness (cost per point improvement in the Paediatric Quality of Life Inventory (PedsQL)) and cost-utility (cost per quality-adjusted life year (QALY)) of CYPHP versus EUC also from an NHS and PSS perspective (National Institute for Health and Care Excellence (NICE) reference case ${ }^{21}$ ). Third, a cost-benefit analysis (cost per monetarised unit of parental well-being and children's QALYs) of CYPHP compared with EUC from a societal perspective will be conducted. The cost-benefit analysis will also account for costs falling on parents and schools. The cost-effectiveness of CYPHP compared with EUC beyond the trial duration will be explored with a state-transition model reflecting natural disease progression for each tracer condition. Existing evaluations of interventions to improve outcomes for children with tracer conditions (such as education initiatives) rarely consider effects beyond 3 years, which may result in a partial characterisation of the intervention effects, and as such, this method is a novel application in child health economics research. Both the economic evaluation and the state-transition model are essential as they will determine whether potential health gains related to the intervention justify its costs relative to EUC, and therefore whether a decision to provide and roll out the intervention is justifiable in terms of efficiency.

Both the population and tracer-condition analyses aim to inform decisions on the current CYPHP provision in Lambeth and Southwark and throughout the South East London Integrated Care System, as well as its potential expansion to other areas if proven efficient.

\section{METHODS AND ANALYSIS \\ Study design}

The study design and intervention components are outlined in detail in our published trial protocol paper. ${ }^{18}$ In summary, 70 general practices in Southwark and Lambeth were grouped into 23 virtual clusters, occurring naturally for GP-paediatrician colocated clinics. Twelve of these clusters were assigned to the intervention (CYPHP) and 11 clusters to the control group (EUC). For randomisation, clusters were stratified by borough, and restricted randomisation was carried out to ensure the number of CYP under 16 years, their socioeconomic status (measured by the Index of Multiple Deprivation and Income Deprivation Affecting Children Index) and number of outpatient referrals were similar between the two study arms. The trial population includes CYP under 
Table 1 Key features of the CYPHP intervention and evaluation

$\begin{array}{ll}\begin{array}{l}\text { Targeted recruitment sample } \\ \text { without loss to follow-up }\end{array} & \text { A theoretically informed intervention (theoretical domains framework). } \\ \text { Route to change } & \text { Evidence-based (based on systematic review on integrated care models for child } \\ & \text { health }{ }^{16} \text { ). } \\ \text { Integrates care in line with patient, provider and policy perspectives-providing efficient, } & \text { preventive access to care, closer to home. } \\ \text { Main strengths } & \text { Opportunistic randomised controlled trial. } \\ \text { Sich data with both patient-reported and routine service use data. } & \text { Embedded process evaluation to assess CYPHP implementation success. } \\ \text { Stakeholder involvement } & \text { CYPHP was developed with children and young people, carers, front-line practitioners and } \\ & \text { health service commissioners. }\end{array}$

CYPHP, Children and Young People's Health Partnership.

16 years of age registered to a general practice in Southwark or Lambeth. Key information on the CYPHP intervention and evaluation is summarised in table 1 .

\section{Intervention and control arms}

The study structure and components of CYPHP and EUC are described in figure 1. As the intervention arm provides CYPHP on top of EUC, EUC is delivered at all practices. CYPHP offers universal services (available to all CYP, with any childhood condition) and targeted services (available only to CYP with tracer conditions-asthma, constipation, and/or eczema). EUC is composed of several patient self-management support tools for families and resources available to health providers to provide higher quality and more joined-up care for CYP.

Specifically, CYPHP includes

\section{- CYPHP universal services}

- In-reach clinics, integrated child health clinics codelivered by patch paediatricians and GPs (patch paediatricians are linked to a cluster of general practices) as part of a multidisciplinary CYP health team located in the community.

- Lunch-and-learn sessions, where a multidisciplinary group of CYP health professionals, including paediatricians and primary care staff, share knowledge, review cases, create common professional cultures, build and reinforce team working practices.

CYPHP targeted services (tracer conditions only)

- Specialist nurse-led services, usually delivered by a CYPHP nurse trained in biopsychosocial care (mental health and other specialists are available too if needed) at the CYP's home, during a visit at a community-based clinic or through a phone call or message. It includes health promotion and selfmanagement advice on tracer conditions. Patients are triaged and care is planned based on a preassessment biopsychosocial health check (CYPHP Health Check) and patient records. The CYPHP Health Check is administered to patients with asthma, constipation or eczema. It uses validated questionnaires when possible to measure biopsychosocial health. Children's ongoing conditions are assessed with the Patient-Oriented Eczema Measure ${ }^{22}$ for children with eczema, the Asthma Control Test ${ }^{23}$

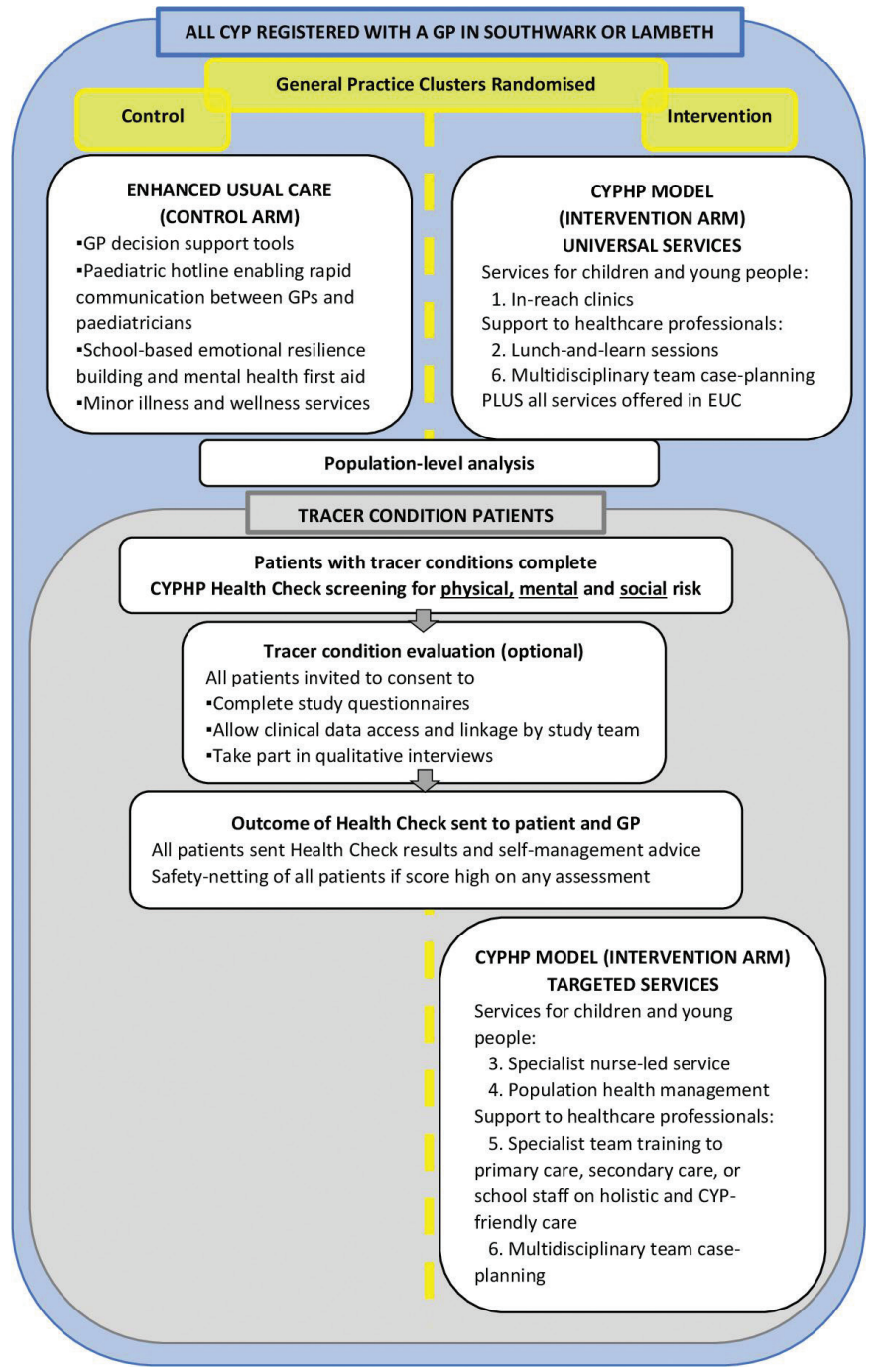

Figure 1 Study population and intervention flow. CYP, children and young people; CYPHP, Children and Young People's Health Partnership; GP, general practitioner. 
for asthma and a bespoke CYPHP constipation questionnaire (validation work under way). The Strengths and Difficulties Questionnaire ${ }^{24}$ is used as an emotional and behavioural screening questionnaire. Finally, a set of bespoke social questions to understand a family's broader situation and factors that may affect their health and care, such as financial worries and days lost of school or work, is also included. Participants who consent as research subjects do also complete the PedsQL ${ }^{25}$ and the Child Health Utility 9 Dimensions (CHU-9D). ${ }^{26}$

- Population health management, where CYP with tracer conditions are sent text messages and a letter from their GP, encouraging them to participate in early intervention and care. Recipients are identified based on analyses of electronic health records and actively reached out to connect them with the healthcare system and to improve the management of their conditions before they exacerbate.

- Specialist team training, including education and training for primary care, secondary care or school staff on evidence-based, holistic and CYP-friendly care for tracer conditions, is delivered by CYPHP professionals.

Multidisciplinary team case planning is important for CYPHP delivery, present in both universal and targeted services. It includes case planning and both formal and informal education and training for professionals providing CYPHP.
Table 2 describes the expected inputs, frequency and duration of each CYPHP component. All these data elements will be collected, as actual implementation may differ from protocolised implementation.

CYP access universal services through referrals from their paediatrician or GP. For specialist services, entry sources include direct referrals (from GP, paediatrician, school nurse or emergency department); self-referrals (availability publicised through community events and posters in GP practices); and proactive case finding (CYP with tracer conditions are sent text messages and a letter from their GP). Further details on CYPHP's implementation are included in the publicly available handbook. ${ }^{27}$

\section{Patient and public involvement}

Stakeholders were involved in the development of the theoretical framework for CYPHP, identification of research questions and refining the research methodology. Stakeholders included CYP, carers, front-line practitioners and health service commissioners. A patient and public involvement group was developed with children and their families and was consulted with regard to evaluation design, including appropriateness of outcome measures and consent procedures.

\section{Economic evaluation within the trial}

Population-level cost analysis

The goal of the population-level analysis is to assess the impact of CYPHP compared with EUC on healthcare costs of health service use. This analysis will use

Table 2 Protocolised inputs, frequency and duration of CYPHP components

\begin{tabular}{|c|c|c|c|c|}
\hline Intervention component & Inputs & Frequency & Duration & Comments \\
\hline 1. In-reach clinics & $\begin{array}{l}\text { Labour: GP and patch } \\
\text { paediatrician }\end{array}$ & Once a month & $\begin{array}{l}20-30 \text { min per } \\
\text { patient }\end{array}$ & 2-3 hours total \\
\hline $\begin{array}{l}\text { 4. Population health } \\
\text { management }\end{array}$ & $\begin{array}{l}\text { Labour: population health } \\
\text { clinician, analyst and } \\
\text { manager }\end{array}$ & Varies & Varies & $\begin{array}{l}\text { Data: access, storage, } \\
\text { analysis } \\
\text { Proactive case finding: } \\
\text { costs for sending } \\
\text { messages }\end{array}$ \\
\hline $\begin{array}{l}\text { 6. Multidisciplinary team } \\
\text { case planning }\end{array}$ & $\begin{array}{l}\text { Labour: CYPHP nurse, } \\
\text { mental health specialist, } \\
\text { paediatrician and GP who } \\
\text { works alongside CYPHP }\end{array}$ & Once a week & $60 \mathrm{~min}$ & . \\
\hline
\end{tabular}

CYPHP, Children and Young People's Health Partnership. 
the whole study population, which includes CYP, 0-15 years of age, registered with a Southwark or Lambeth GP practice. Health service use will include primary care consultations, visits with paediatricians, and hospital outpatient, hospital inpatient, and A\&E care during 6 and 12 months. Patient-level costs will be obtained by multiplying unit costs by use. National unit costs for children's services will be obtained from the Unit Costs of Health and Social Care 2019/20 by the Personal Social Services Resource Unit ${ }^{28}$ and the NHS reference costs for 2015-2016. ${ }^{29}$ Due to the often-skewed cost distribution with a large number of zeros and a long right-hand tail, the modified Park Test and Pregibon Link test will assess the most appropriate distribution and link to calibrate a generalised linear model (GLM) for costs, for example, with a gamma distribution and a log link. ${ }^{30} 31$ The cost model will adjust for a binary variable indicating whether the children or young person belonged to the intervention or control arm and any demographic variables that show imbalance between the two groups.

\section{Tracer conditions: cost-effectiveness/utility and cost-benefit analyses}

This within-trial economic evaluation will also compare CYPHP with EUC for patients under 16 with asthma, constipation and/or eczema. Three types of economic evaluation will be conducted. The costeffectiveness analysis, using point improvement in the PedsQL scale as the primary outcome, and the cost-utility analysis, based on QALYs from the CHU9D, will adopt an NHS and PSS perspective. The costbenefit analysis will take a societal perspective and value parental well-being with the Warwick-Edinburg Mental Well-being Scale (WEMWBS). These analyses will adhere to guidelines for conducting economic evaluations alongside clinical trials and the most recent NICE public health reference case. ${ }^{32-35}$

Costing: identification, measurement and valuation of resources Costing involves identifying, measuring and valuing the resources used to deliver and participate in the intervention and consequential health and social services use. In a complex system change such as CYPHP, the comprehensive identification of resources requires close collaboration with the implementation and the process evaluation teams.

\section{Identification of resource use}

From an NHS and PSS perspective, resources used relate to the delivery of the intervention and health and social care use by patients (table 3 ). Intervention delivery mostly includes time spent by medical professionals and service managers delivering CYPHP services. From a societal perspective, time spent by school staff participating in CYPHP and time away from work or school by parents and CYP are also accounted for. Because both intervention and control practices include EUC, EUC's delivery costs will be disregarded. Service use and time away from school and work will be considered for both CYPHP and EUC.

\section{Measurement of resource use}

Resources used to implement CYPHP will be gathered from seven data sources, including the study's accounting data, service caseloads, CYPHP nurse's personal caseload notes, study questionnaires, primary care data, secondary care data and interviews with CYPHP nurses (table 3). EMIS will provide location, type, number and length of consultations part of in-reach and specialist team services. CYPHP nurse's caseload notes will supply information on specialist team training and multidisciplinary team case planning. Time spent at lunch-and-learn sessions will be obtained from service caseloads. Patient-level service use will be gathered from primary and secondary care activity files. Family and CYP time away from work or school are questions included in the study questionnaires. Interviews with a random sample of CYPHP nurses to understand their phone usage and transportation to patient visits will also be conducted.

\section{Valuation of resource use}

As with the population-level cost analysis, national unit costs for children's services will be obtained from the PSSRU $^{28}$ and NHS reference costs for 2015-2016. ${ }^{29}$ The Unit Costs of Health and Social Care V.2014 will also be used to value referrals to social care services. ${ }^{36}$ Unit costs not available from these sources will be collected from trial records directly (eg, monthly rent of children's health centre use). All unit costs will be presented in pounds sterling $(£)$ for a base cost year 2019/2020; the NHS Cost Inflation Index will be used to adjust for inflation. ${ }^{36}$ As the horizon of the withintrial analysis is 6 and 12 months, no discounting will be applied to either costs or outcomes.

\section{Computation of total costs}

Total costs will be computed at the patient level by summing intervention delivery costs (only CYP in intervention arm) and health service use cost (CYP in intervention and control arms):

- Intervention delivery costs will include set-up, CYPHP delivery and overhead costs. Some of these components will vary across patients $(\mathrm{eg}$, specialist team services); others across clinics (eg, staff specialist training); and others will be the same for all patients (eg, overhead costs such as the cost of administration and facilities). Staff specialist training costs, costs of universal services (in-reach clinics), intervention set-up costs and overheads will each be apportioned. CYP with tracer conditions are the target population of the economic evaluation. The cost of universal services, however, also needs to be considered as CYP with tracer conditions may be referred to specialist team 
Table 3 Identification and measurement of costs

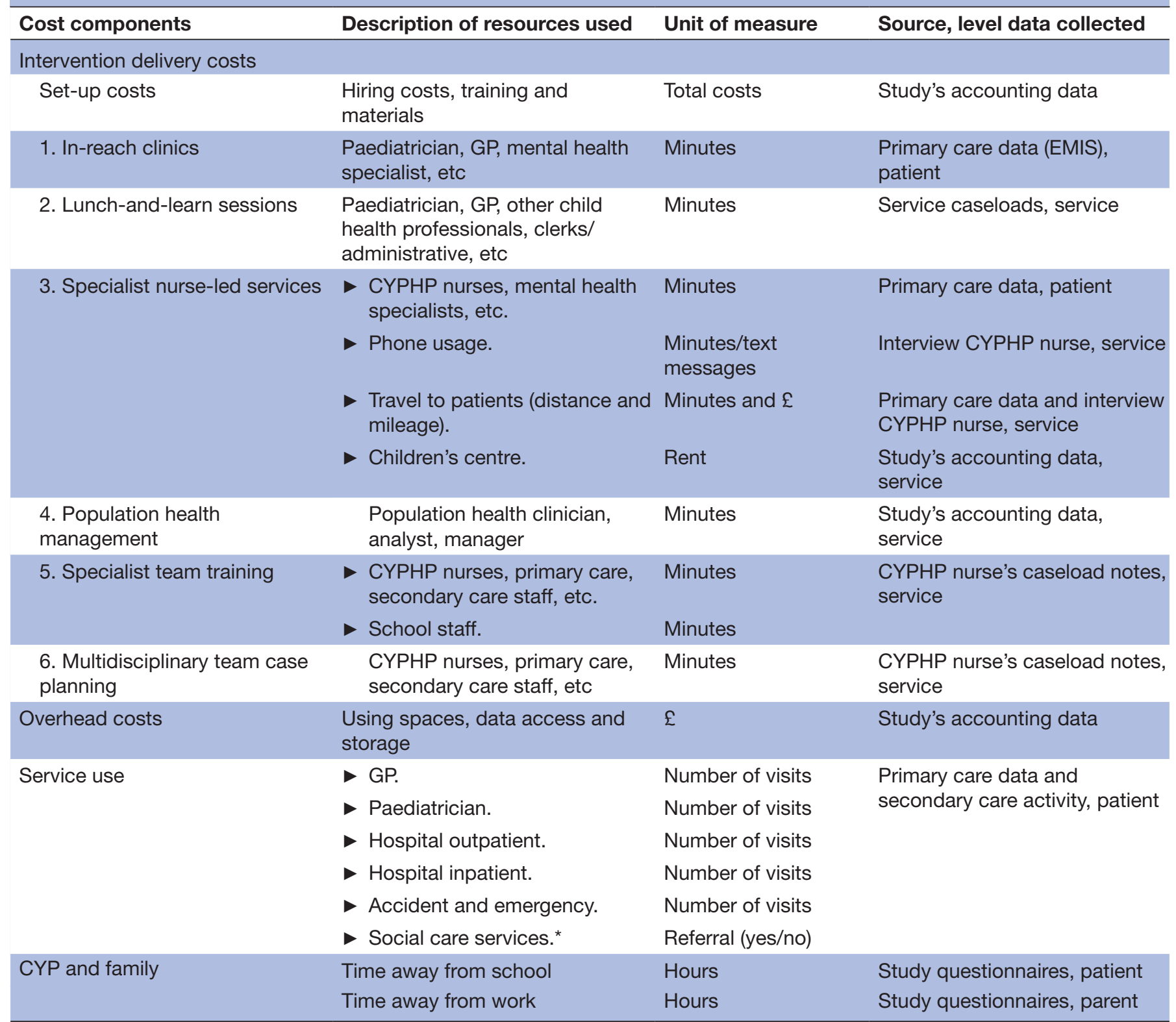

${ }^{*}$ CYPHP nurses may refer CYP and their families to social care services. An indicator for referrals to social services is available in primary care data. Secondary care data (inpatient stays, A\&E attendances and outpatient visits) will be obtained from Guy's and Saint Thomas' NHS Foundation Trust and King's College Hospital data.

CYP, children and young people; CYPHP, Children and Young People's Health Partnership; EMIS, Egton Medical information Systems; GP, general practitioner.

services during an in-reach clinic visit. Different apportioning rules will be used; for example, the costs of universal services could be apportioned by using the percentage of CYP with tracer conditions who were referred by in-reach clinics. Total perpatient apportioned costs will be added to patientlevel specialist team services costs.

- Health service use costs will result from multiplying the quantity of services used by their unit cost and summing across service types for each patient.

Total costs of patients in the control arm will only reflect health service use costs.
In the cost-benefit analysis, total costs will also include costs borne by patients, parent and schools. Patient and parents' costs will be composed of school and work time lost, respectively. Schools' costs will include time spent by school staff attending specialist team training.

Measurement and valuation of health outcomes

The trial's primary health outcome measure of the tracer-condition evaluation is the PedsQL, which will be used in the cost-effectiveness analysis. The PedsQL includes 23 items covering physical, emotional, social 
and school functioning ${ }^{25}$ and is available through six age-specific questionnaires (0-12 months, 13-24 months, 2-4 years, 5-7 years, 8-12 years and 13-17 years). The PedsQL has been shown to be reliable, valid and responsive to meaningful change across general and disease-specific populations. ${ }^{25} 3738$ The CHU-9D-a generic preference-based measure of paediatric health-related quality of life that allows the calculation of QALYs-will be the health outcome measure for cost-utility analysis. The nine items of CHU-9D cover feeling worried, sad, tired and annoyed; perceptions of schoolwork; sleep; daily routine; and social activities. The tool is designed to be administered to CYP between 7 and 17 years of age, and a proxy version to be completed by parents is available for younger children. ${ }^{39}{ }^{40}$ The WEMWBS ${ }^{41}$ will serve as a well-being questionnaire for parents. All questionnaires were administered at baseline and at two follow-up points (6 and 12 months). Questionnaires completed during the first phase of the COVID-19 pandemic (12 March-6 July 2020) will be repeated after this period, and follow-up measures will be delayed.

In the cost-benefit analysis, QALYs and WEMWBS will be combined by converting both to pound sterling values. QALYs will be monetised by using the government sector willingness-to-pay of $£ 20000$ to $£ 30000$ per QALY gained. ${ }^{42}$ For WEMWBS, the monetary values published by Simetrica and Housing Associations' Charitable Trust (HACT) for each Short-Version WEMWBS (SWEMWBS) score will be employed and converted to cost year 2019/20. ${ }^{43}$ The SWEMWBS score can be obtained from the original WEBWMS using 7 of its 14 statements about thoughts and feelings.

\section{Statistical analyses}

The intention-to-treat population will be used in statistical analyses. First, differences between protocolised and actual intervention components (including inputs, frequency and duration of each component) will be assessed (table 2). Second, univariate analyses will be conducted to describe sample mean differences and variability across time between treatment and control groups for each outcome. Three time points will contribute to analysis: baseline, 6 months and 12 months. Third, to adjust for treatment group imbalances, four multilevel regression models will be estimated; one each for total costs, QALYs, PedsQL score and monetary benefits ( $£$ corresponding to QALYs and WEBWMS scores together). ${ }^{44}$ Each model will include a variable indicating participation in intervention or control and variables that, despite randomisation, may still be unequally distributed between intervention and control groups such as age, gender and deprivation level for the patient-level models. For the regression model predicting QALYs, the baseline QALYs will also be controlled for. ${ }^{45}$ Benefits will be estimated using ordinary least squares, and costs will be obtained with a GLM model with a gamma distribution and a log link. Both the use of a GLM and limited dependent variable mixture models will be considered when modelling QALYs. ${ }^{46}$ All models will cluster SEs to account for correlation of patients in the same CYPHP cluster.

For each outcome variable and intervention and control groups separately, mean predicted values will be generated. Three incremental cost-effectiveness ratios (difference between intervention and control in mean predicted costs over difference in mean predicted outcomes) will be computed, one for the cost-effectiveness analysis (based on PedsQL scores), another for the cost-utility analysis (using QALYs) and a third one for the cost-benefit analysis $(£)$. These three incremental cost-effectiveness ratios (ICERs) will be generated based on 6 and 12 months data.

The pattern and amount of missing data between treatment and control groups by study variable will be assessed. If data are missing completely at random for both treatment and control groups and the percentage of missing data is below $5 \%$, missing data will be ignored. If data are missing at random (MAR), multiple imputation accounting for clustering (such as fixed effects) will be used. ${ }^{47}$ When the data are MAR, multiple imputation can lead to consistent, asymptotically efficient and asymptotically normal estimates. ${ }^{48}$

\section{Handling uncertainty}

The level of decision uncertainty arising from sampling and assumptions on key parameter estimates with policy impact will be assessed. CIs for ICERs based on the nonparametric bootstrap method will be generated, ${ }^{49}$ along with acceptability curves to reflect the probability of CYPHP being cost-effective as the willingness-to-pay per QALY (or other health outcome) increases. Deterministic sensitivity analyses on chosen variables (such as intervention set-up costs, intensity of services delivered and social care costs) will assist in identifying key drivers of the results. Subgroup analysis of cost-effectiveness results by tracer condition and quintiles of the Index of Multiple Deprivation (IMD) will be conducted as long as a sufficient sample size is available.

\section{Long-term modelling of health and costs beyond the trial}

A state-transition model reflecting natural disease progression will be developed for each tracer condition to predict the cost-effectiveness of CYPHP compared with EUC beyond the trial duration. Trial data will be used to define the health states and transition probabilities among states, and to calculate the costs and effects from an NHS/PSS perspective. Existing literature and publicly available statistics (eg, Office of National Statistics and existing UK cohort studies) will also be used to gather transition probabilities across states beyond 12 months. A functional form characterising the sustainability of intervention effects into the longer run (changes in healthrelated quality of life and health service use) will be inferred based on 6 and 12 months of trial data. The effect of alternative analytical horizons on the cost-effectiveness 
of CYPHP versus EUC will be tested in sensitivity analyses, including 2, 5 and 10 years.

\section{DISCUSSION}

The CYPHP Evelina London model is a health-systems strengthening programme to advance towards integrated and high-quality care for CYP in the UK. By offering universal and targeted services, CYPHP aims to overcome patient-level and provider-level barriers to effective management of physical and mental health and to foster optimal health behaviour. The aims of this economic evaluation are to establish the impact of CYPHP on healthcare costs at the population level and the cost-effectiveness of the intervention among CYP with tracer conditions. Asthma, constipation and eczema serve as examples of common long-term conditions among CYP. Lessons from managing these conditions should inform a broader health system response to the epidemiological transition to chronic diseases.

\section{Strengths and weaknesses}

Beyond temporary trial suspension, COVID-19 may have affected our study in at least two ways. First, CYPHP delivery may not return to normal after the pandemic. Differences in the frequency and duration of each CYPHP component before and after COVID-19 will be assessed in sensitivity analyses. Second, some follow-up questionnaires were due during COVID-19. When possible, data were collected, and an additional data point after COVID-19 was included for these participants to isolate changes in health status due to the pandemic. Besides the effects of COVID-19, the intensity of services delivered as part of CYPHP may not be fully standardised across GP practices. Variability in service intensity across practices and its impact on cost-effectiveness results will be assessed in sensitivity analyses. Additionally, health utility outcome measurement for children below 5 may lack reliability as the questionnaire has not been psychometrically tested for this younger age group. ${ }^{2650}$ This measurement challenge will be addressed by using multiple economic evaluation perspectives and health outcomes (such as the PedsQL) to provide a comprehensive and transparent assessment of the effects of the intervention.

By carrying out three economic evaluations (costeffectiveness, cost-utility and cost-benefit) under two different perspectives (NHS and PSS, and societal), we aim to inform stakeholders with various interests, including clinical commissioning groups and evolving integrated care system, GP federations, provider trusts, CYP and their families. With CYPHP, healthcare use costs may remain stable if primary care visits increase, but hospitalisations and emergency room visits decrease. Parents and children's costs related to time lost from work or school are also expected to decline with CYPHP if CYP's tracer conditions are well managed. Our planned analyses will allow both to be studied and accounted for.

The long-term model will assess the cost-effectiveness of CYPHP compared with EUC beyond the trial duration to fully capture intervention effects on children with asthma, constipation and/or eczema. Existing cost-effectiveness studies assessing interventions for CYP with these tracer conditions rarely include a long-term model, and the duration of RCTs of education, coaching, nurse-led clinics or treatments for the tracer conditions tend to be under 3 years. ${ }^{51-56}$ CYPHP is expected to foster long-lasting improvements beyond 12 months in health outcomes due to changes in disease management behaviour among the CYP and family, and also health professionals. The natural progression of the tracer conditions indicates that a substantial percentage of children continue to experience symptoms beyond 12 months, and sometimes even into adulthood. Asthma in childhood persists into adulthood for $79 \%$ of the cases. ${ }^{57}$ About half of children with atopic eczema still have the problem as adults. ${ }^{589}$ Twenty-five per cent of children with functional constipation continue to experience symptoms as adults. ${ }^{6061}$

This study will contribute rigorous evidence about health economics of children's integrated healthcare in the UK, where there has been a notable paucity of high-quality studies. Results from this study will directly inform decisions on children's healthcare provision in South East London and will provide rigorous evidence to support policy nationally and internationally.

\section{ETHICS AND DISSEMINATION}

Ethics approval was obtained from South WestCornwall and Plymouth Research Ethics Committee. Results will be submitted for publication in peerreviewed journals, made available in briefing papers for local decision-makers, and provided to the local community through website and public events. Findings will be generalisable to community-based models of care, especially in urban settings.

\section{Author affiliations}

${ }^{1}$ Department of Population Health Sciences, King's College London, London, UK ${ }^{2}$ School of Women's and Children's Health, University of New South Wales Faculty of Medicine, Sydney, New South Wales, Australia

${ }^{3}$ Department of Psychological Interventions, University of Surrey, Guildford, UK ${ }^{4}$ Department of Women's and Children's Health, King's College London, London, UK ${ }^{5}$ Population Health Sciences, King's College London, London, UK

${ }^{6}$ NIHR Biomedical Research Centre at Guy's and Saint Thomas' NHS Foundation Trust and King's College, London, UK

\section{Twitter Marina Soley-Bori @MarinaSoleyBori}

Contributors MS-B: conceptualisation; methodology; writing, original draft; writing, review and editing; investigation; visualisation. RL: writing, review and editing; funding acquisition. R-MS: writing, review and editing; investigation. JF and EC: writing, review and editing. JF-R: conceptualisation; methodology; supervision; 
writing, review and editing. IW: writing, review and editing; funding acquisition. JF-R and IW are last authors.

Funding This work was supported by Guy's and St Thomas' Charity.

Competing interests None declared.

Patient and public involvement Patients and/or the public were involved in the design, conduct, reporting or dissemination plans of this research. Refer to the Methods and analysis section for further details.

Patient consent for publication Not applicable.

Provenance and peer review Not commissioned; externally peer reviewed.

Open access This is an open access article distributed in accordance with the Creative Commons Attribution Non Commercial (CC BY-NC 4.0) license, which permits others to distribute, remix, adapt, build upon this work non-commercially, and license their derivative works on different terms, provided the original work is properly cited, appropriate credit is given, any changes made indicated, and the use is non-commercial. See: http://creativecommons.org/licenses/by-nc/4.0/.

\section{ORCID iDs}

Marina Soley-Bori http://orcid.org/0000-0002-8348-3575

Lizzie Cecil http://orcid.org/0000-0002-5281-6656

\section{REFERENCES}

1 Cheung R. International comparisons of health and wellbeing in early childhood. London Nuffield Trust, 2018.

2 OECD. Health Status: Maternal and infant mortality [Internet] Available: https://stats.oecd.org/index.aspx?queryid=30116\# [Accessed 17 Sep 2020].

3 Wolfe I, Thompson M, Gill P, et al. Health services for children in Western Europe. Lancet 2013;381:1224-34.

4 Wolfe I, Macfarlane A, Donkin A. Why children die: death in infants, children, and young people in the UK-Part a. Lond R Coll Paediatr Child Health, 2014.

5 Davies SC, Lemer C, Strelitz J, et al. Our children deserve better: prevention pays. Lancet 2013;382:1383-4.

6 Hoey H, Aanstoot HJ, Chiarelli F, et al. Good metabolic control is associated with better quality of life in 2,101 adolescents with type 1 diabetes. Diabetes Care 2001;24:1923-8.

7 Sundbom F, Malinovschi A, Lindberg E, et al. Effects of poor asthma control, insomnia, anxiety and depression on quality of life in young asthmatics. J Asthma 2016;53:398-403.

8 Boyd M, Lasserson TJ, McKean MC, et al. Interventions for educating children who are at risk of asthma-related emergency department attendance. Cochrane Database Syst Rev 2009;2:CD001290.

9 Ordoñez GA, Phelan PD, Olinsky A, et al. Preventable factors in hospital admissions for asthma. Arch Dis Child 1998;78:143-7.

10 Castro M, Zimmermann NA, Crocker S, et al. Asthma intervention program prevents readmissions in high healthcare users. Am J Respir Crit Care Med 2003;168:1095-9.

11 Nunes C, Ladeira S, Asthma LS. Asthma, from childhood to adulthood: a prospective 20-year longitudinal study of a cohort of asthmatics. J Investig Allergol Clin Immunol 2002;12:242-9.

12 Stevens CA, Turner D, Kuehni CE, et al. The economic impact of preschool asthma and wheeze. Eur Respir J 2003;21:1000-6.

13 UNICEF. Convention on the Rights of the Child [Internet]. Available: https://www.unicef.org/child-rights-convention [Accessed 03 Oct 2020]

14 Belli PC, Bustreo F, Preker A. Investing in children's health: what are the economic benefits? Bull World Health Organ 2005;83:777-84.

15 Guyer B, Ma S, Grason H. INVESTMENTS TO PROMOTE CHILDREN 'S HEALTH: A systematic literature review and economic analysis of interventions in the preschool period. Baltim MD Johns Hopkins Bloom Sch Public Health, 2008.

16 Wolfe I, Satherley R-M, Scotney E, et al. Integrated care models and child health: a meta-analysis. Pediatrics 2020;145:e20183747.

17 GOV.UK. 2019 child health profiles [Internet]. Available: https://www. gov.uk/government/statistics/2019-child-health-profiles [Accessed 13 Feb 2020].

18 Newham JJ, Forman J, Heys M, et al. Children and young people's health partnership (CYPHP) Evelina London model of care: protocol for an opportunistic cluster randomised controlled trial (cRCT) to assess child health outcomes, healthcare quality and health service use. BMJ Open 2019;9:e027301.

19 Satherley R-M, Green J, Sevdalis N, et al. The Children and Young People's Health Partnership Evelina London Model of Care: process evaluation protocol. BMJ Open 2019;9:e027302.
20 Tramonti F, Giorgi F, Fanali A. Systems thinking and the biopsychosocial approach: a multilevel framework for patient-centred care. Syst Res Behav Sci 2021;38:215-30.

21 NICE. Guide to the methods of technology appraisal 2013 [Internet], 2013. Available: https://www.nice.org.uk/process/pmg9/chapter/ foreword

22 Charman CR, Venn AJ, Williams HC. The patient-oriented eczema measure: development and initial validation of a new tool for measuring atopic eczema severity from the patients' perspective. Arch Dermatol 2004;140:1513-9.

23 Nathan RA, Sorkness CA, Kosinski M, et al. Development of the asthma control test: a survey for assessing asthma control. J Allergy Clin Immunol 2004;113:59-65.

24 Goodman R. Psychometric properties of the strengths and difficulties questionnaire. J Am Acad Child Adolesc Psychiatry 2001;40:1337-45.

25 Varni JW, Seid M, Rode CA. The PedsQL: measurement model for the pediatric quality of life inventory. Med Care 1999;37:126-39.

26 Furber G, Segal L. The validity of the child health utility instrument (CHU9D) as a routine outcome measure for use in child and adolescent mental health services. Health Qual Life Outcomes 2015;13:22.

27 Institute for Women and Children's Health, King's Health Partners, London, UK. Children and Young People's CHILDS (Children's Health Integrated Care and Learning Delivery System) framework. [Internet]. Available: childsframework.org

28 PSSRU. Unit Costs of Health and Social Care 2020 [Internet], 2020. Available: https://www.pssru.ac.uk/project-pages/unit-costs/unitcosts-2020/

29 GOV.UK. NHS reference costs 2015 to 2016 [Internet]. Available: https://www.gov.uk/government/publications/nhs-reference-costs2015-to-2016 [Accessed 21 May 2020]

30 Franklin M, Lomas J, Walker S, et al. An educational review about using cost data for the purpose of cost-effectiveness analysis. Pharmacoeconomics 2019;37:631-43.

31 Mantopoulos T, Mitchell PM, Welton NJ, et al. Choice of statistical model for cost-effectiveness analysis and covariate adjustment: empirical application of prominent models and assessment of their results. Eur J Health Econ 2016;17:927-38.

32 NICE. Methods for the development of NICE public health guidance (third edition, 2013

33 Glick HA, Doshi JA, Sonnad SS. Economic evaluation in clinical trials. OUP Oxford, 2014.

34 Fox-Rushby J, Cairns J. Economic evaluation. McGraw-Hill Education (UK), 2005.

35 Ramsey SD, Willke RJ, Glick H, et al. Cost-Effectiveness analysis alongside clinical trials II-An ISPOR good research practices Task force report. Value Health 2015;18:161-72.

36 Curtis L. Unit costs of health and social care. Personal Social Services Research Unit: University of Kent, 2014

37 Chan KS, Mangione-Smith R, Burwinkle TM, et al. The PedsQL: reliability and validity of the short-form generic core scales and asthma module. Med Care 2005;43:256-65.

38 Seid M, Limbers CA, Driscoll KA, et al. Reliability, validity, and responsiveness of the pediatric quality of life inventory (PedsQL) generic core scales and asthma symptoms scale in vulnerable children with asthma. J Asthma 2010;47:170-7.

39 Stevens K. Developing a descriptive system for a new preferencebased measure of health-related quality of life for children. Qual Life Res 2009;18:1105-13.

40 Stevens K. Assessing the performance of a new generic measure of health-related quality of life for children and refining it for use in health state valuation. Appl Health Econ Health Policy 2011;9:157-69.

41 Tennant R, Hiller L, Fishwick R, et al. The Warwick-Edinburgh mental well-being scale (WEMWBS): development and UK validation. Health Qual Life Outcomes 2007;5:63.

42 NICE. 6 Incorporating health economics | Methods for the development of NICE public health guidance (third edition) Guidance | NICE [Internet]. NICE. Available: https://www.nice.org.uk/ process/pmg4/chapter/incorporating-health-economics [Accessed 02 Mar 2020]

43 Trotter L, Rallings Adams M-K. Valuing improvements in mental health: applying the wellbeing valuation method to WEMWBS. HACT, 2017.

44 Gomes M, Grieve R, Nixon R, et al. Methods for covariate adjustment in cost-effectiveness analysis that use cluster randomised trials. Health Econ 2012;21:1101-18.

45 Manca A, Hawkins N, Sculpher MJ. Estimating mean QALYs in trialbased cost-effectiveness analysis: the importance of controlling for baseline utility. Health Econ 2005;14:487-96. 
46 Hernández Alava M, Wailoo AJ, Ara R. Tails from the peak district: adjusted limited dependent variable mixture models of EQ-5D questionnaire health state utility values. Value Health 2012:15:550-61.

47 DiazOrdaz K, Kenward MG, Gomes M, et al. Multiple imputation methods for bivariate outcomes in cluster randomised trials. Stat Med $2016 ; ; 35: 3482-96.10$.

48 Allison PD. Missing Data [Internet. SAGE Publications Inc, 2020. https://us.sagepub.com/en-us/nam/missing-data/book9419

49 Chaudhary MA, Stearns SC. Estimating confidence intervals for costeffectiveness ratios: an example from a randomized trial. Stat Med 1996;15:1447-58.

50 Frew EJ, Pallan M, Lancashire E, et al. Is utility-based quality of life associated with overweight in children? Evidence from the UK waves randomised controlled study. BMC Pediatr 2015;15:10.1186/s12887015-0526-1.

51 Sullivan SD, Weiss KB, Lynn $\mathrm{H}$, et al. The cost-effectiveness of an inner-city asthma intervention for children. J Allergy Clin Immunol 2002;110:576-81.

52 Madge P, McColl J, Paton J. Impact of a nurse-led home management training programme in children admitted to hospital with acute asthma: a randomised controlled study. Thorax 1997;52:223-8.

53 Noyes K, Bajorska A, Fisher S, et al. Cost-Effectiveness of the school-based asthma therapy (SBAT) program. Pediatrics 2013;131:e709-17.
54 Franco R, Santos AC, do Nascimento HF, et al. Cost-effectiveness analysis of a state funded programme for control of severe asthma. BMC Public Health 2007;7:82.

55 Schuttelaar MLA, Vermeulen KM, Coenraads PJ. Costs and cost-effectiveness analysis of treatment in children with eczema by nurse practitioner vs. dermatologist: results of a randomized, controlled trial and a review of international costs. $\mathrm{Br} J$ Dermatol 2011;165:10.1111/j.1365-2133.2011.10470.x:600-11.

56 Ismail N, Ratchford I, Proudfoot C, et al. Impact of a nurse-led clinic for chronic constipation in children. $J$ Child Health Care 2011;15:10.1177/1367493511406568:221-9.

57 Andersson M, Hedman L, Bjerg A, et al. Remission and persistence of asthma followed from 7 to 19 years of age. Pediatrics 2013;132:e435-42.

58 Abuabara K, Hoffstad O, Troxel A, et al. Atopic dermatitis disease control and age: a cohort study. J Allergy Clin Immunol 2015;136:190-2.

59 Ridd MJ, King AJL, Le Roux E, et al. Systematic review of selfmanagement interventions for people with eczema. $\mathrm{Br} J$ Dermatol 2017;177:719-34.

60 Auth MKH, Vora R, Farrelly P, et al. Childhood constipation. BMJ 2012;345:e7309.

61 Bongers MEJ, van Wijk MP, Reitsma JB, et al. Long-term prognosis for childhood constipation: clinical outcomes in adulthood. Pediatrics 2010;126:e156-62. 